\title{
Behavorial effects in individual decisions of network formation
}

Citation for published version (APA):

Harmsen - van Hout, M. J. W., Dellaert, B. G. C., \& Herings, P. J. J. (2008). Behavorial effects in individual decisions of network formation. METEOR, Maastricht University School of Business and Economics. METEOR Research Memorandum No. 019 https://doi.org/10.26481/umamet.2008019

Document status and date:

Published: 01/01/2008

DOI:

10.26481/umamet.2008019

Document Version:

Publisher's PDF, also known as Version of record

\section{Please check the document version of this publication:}

- A submitted manuscript is the version of the article upon submission and before peer-review. There can be important differences between the submitted version and the official published version of record.

People interested in the research are advised to contact the author for the final version of the publication, or visit the DOI to the publisher's website.

- The final author version and the galley proof are versions of the publication after peer review.

- The final published version features the final layout of the paper including the volume, issue and page numbers.

Link to publication

\footnotetext{
General rights rights.

- You may freely distribute the URL identifying the publication in the public portal. please follow below link for the End User Agreement:

www.umlib.nl/taverne-license

Take down policy

If you believe that this document breaches copyright please contact us at:

repository@maastrichtuniversity.nl

providing details and we will investigate your claim.
}

Copyright and moral rights for the publications made accessible in the public portal are retained by the authors and/or other copyright owners and it is a condition of accessing publications that users recognise and abide by the legal requirements associated with these

- Users may download and print one copy of any publication from the public portal for the purpose of private study or research.

- You may not further distribute the material or use it for any profit-making activity or commercial gain

If the publication is distributed under the terms of Article $25 \mathrm{fa}$ of the Dutch Copyright Act, indicated by the "Taverne" license above, 
Marjolein J.W. Harmsen-van Hout,

Benedict G.C. Dellaert, P. Jean-Jacques Herings

Behavorial Effects in Individual Decisions of Network Formation

$\mathrm{RM} / 08 / 019$

JEL code: A14, C91, D85

\section{METEबrR}

Maastricht research school of Economics of TEchnology and ORganizations

Universiteit Maastricht

Faculty of Economics and Business Administration P.O. Box 616

NL - 6200 MD Maastricht

phone : ++31433883830

fax : ++31433884873 


\title{
Behavioral Effects in Individual Decisions of Network Formation
}

\author{
Marjolein J.W. Harmsen - van Hout ${ }^{1} \quad$ Benedict G.C. Dellaert ${ }^{2}$ \\ P. Jean-Jacques Herings ${ }^{3}$
}

June 19, 2008

${ }^{1}$ Department of Marketing, Faculty of Economics and Business Administration, Maastricht University, P.O. Box 616, 6200 MD Maastricht, The Netherlands, e-mail: m.vanhout@mw.unimaas.nl, fax: +31 433884918 , phone: +31 433883839

${ }^{2}$ Department of Business Economics, Marketing Section, School of Economics and Business Economics, Erasmus University, P.O. Box 1738, 3000 DR Rotterdam, The Netherlands, e-mail: dellaert@few.eur.nl, fax: +31 10408 9169, phone: +31 104081353

${ }^{3}$ Department of Economics, Faculty of Economics and Business Administration, Maastricht University, P.O. Box 616, 6200 MD Maastricht, The Netherlands, e-mail: p.herings@algec.unimaas.nl, fax: +31 433884878 , phone: +31 433883824 ; +31 433883636 This author would like to thank the Netherlands Organisation for Scientific Research (NWO) for financial support. 


\begin{abstract}
Network formation constitutes an important part of many social and economic processes, but relatively little is known about how individuals make their linking decisions. This article provides an experimental investigation of behavioral effects in individual decisions of network formation. Our findings demonstrate that individuals systematically simplify more complex components of network payoff in their linking decisions. Specifically, they focus on only part of the normative payoff, namely on their own direct payoff and tend to ignore indirect payoff and payoff for others in the network. Additionally, individuals use descriptive behavioral traits of link choice alternatives to guide their choices. They are sensitive to whether an alternative involves link deletion or creation and whether it concerns an isolated or a central node. Furthermore, we find that complexity of one type can moderate individuals' dealing with a complex feature of another type. These behavioral effects have important implications for researchers and managers working in areas that involve network formation.
\end{abstract}

JEL Classification: A14, C91, D85

Keywords: network formation, individual decision making, heuristics, network complexity, choice experiments, mixed logit 


\section{Introduction}

Network formation among individuals is an important phenomenon in many social and economic contexts, ranging from word-of-mouth communications among consumers (e.g., Iacobucci and Hopkins 1992) and social structure (e.g., Granovetter 1995) to intra-organization communication (e.g., Van den Bulte and Moenaert 1998) and virtual communities (e.g., Wellman et al. 1996).

There exists a recent and increasing literature in which researchers experimentally investigate the network formation process. One stream in this literature is involved with testing integral gametheoretical models of network formation. They include variants of Bala and Goyal's (2000) noncooperative network formation model (e.g., Berninghaus et al. 2006, Callander and Plott 2005), Jackson and Wolinsky's (1996) pairwise cooperative network formation model (e.g., Deck and Johnson 2004), and fully cooperative network formation models like Jackson and Van den Nouweland's (2005) (e.g., Charness and Jackson 2006). This research identifies several conditions under which theoretically stable network structures are reproduced in the laboratory and addresses these networks' efficiency. Another stream of experimental studies examines the role of network formation as endogenously emerging in other relevant settings of cooperative decision making (e.g., Brown et al. 2004, Corbae and Duffy 2007, Hauk and Nagel 2001, Kirchsteiger et al. 2005). This research shows that cooperation decisions are considerably influenced when individuals are allowed to choose their partners versus when an interaction structure is imposed.

A common factor in this previous empirical investigation of the network formation process is that individual benefits and costs are given by a payoff function tailored to the specific game-theoretical setting. Therefore, an issue that has been largely ignored is that the complexity of the network formation decisions that individuals face may cause errors or simplifications in their choice process. Although previous research acknowledged the mere existence of errors (e.g., McKelvey and Palfrey 1995, Charness and Jackson 2006), the underlying process remains undisclosed. The objective of the current paper is to investigate such behavioral shifts in individual decision making in networks as a function of typical network complexity features. In particular, we examine whether individuals use simplifying heuristics in dealing with network complexity, similarly to how they use simplifying heuristics in previously studied choice contexts (Johnson and Payne 1985).

We perform an individual decision making experiment in which we vary three complexity features that are relevant in the context of network formation, i.e., structural complexity of the network, transferability of value in the network, and social tradeoff between one's own payoff and others' 
payoff. These properties complicate the choices that individuals make about creating and maintaining links in the network. We examine whether they affect individuals' use of simplifying heuristics. We investigate whether complex network features are represented in a simplified form in the individual decision process, and how complexity of one type can moderate the individual handling of a complex feature of another type.

In general, we investigate whether individuals cope with complexity by attribute substitution, i.e., assessing the target attribute of a judgment object by substituting a property of the object that is more accessible, i.e., comes more readily to mind (Gilovich et al. 2002, Kahneman 2003). Individuals may thus simplify the network formation decision process in two ways. First, they substitute the exact payoff value of a link choice alternative by descriptive attributes that are related to this value, e.g., whether a linking alternative involves link deletion or creation and whether it concerns an isolated or a central node. Second, they focus relatively strongly on the value they derive from their direct neighbors rather than indirect neighbors and they put less weight on the payoffs that others receive. We examine whether these two types of heuristics are more heavily used in more complex settings.

In order to test our hypotheses, we confront participants in the lab with multiple static, noninteractive network situations in which they can choose to create or delete one link or to do nothing. Such a network situation constitutes one of the simplest network linking decision contexts, which allows us to study the effects of network complexity under highly controlled experimental conditions. In more complex network tasks the proposed complexity effects are also likely to play a significant role, but it would be much harder to disentangle each component's separate effect.

The participants' choices have a direct impact on their monetary rewards in the experiment and differ with respect to the three complexity factors (structural complexity, value transferability, social tradeoff), leading to different treatments. We perform a comprehensive parametric test of the hypotheses by estimating a mixed (i.e., random parameters) logit model (McFadden 2001, Hensher et al. 2005). This allows us to investigate the impact of important complex network properties on individuals' decisions, while allowing for heterogeneity of the decision makers.

In Section 2, we present our theoretical framework, leading to hypotheses on behavioral effects in network linking decision making that differ from prior predictions based on full rationality. Section 3 describes the experimental design and the approach used for the mixed logit estimations. The results of our experiment and hypotheses tests are reported in Section 4. Section 5 concludes the paper with discussion. 


\section{Theoretical Framework}

The objective of this section is to present our hypotheses about behavioral effects in individual decisions of network formation and compare them to predictions on individual choice behavior underlying the previous experimental network formation literature. After a description of our setting, the predictions based on prior theories are reviewed in Section 2.1 and our hypotheses are presented in Section 2.2.

The focus of our research is to investigate individuals' behavioral response to variations in network complexity. We address the elementary case of single link formation decisions by individuals. Doing so allows us to investigate complexity effects in a tightly controlled yet relevant setting of network formation decisions. To prevent possible confounding effects that do not originate from network complexity but from strategic interaction among individuals, we focus on individual one-period decisions. Thus a typical decision task as we study it would be described as follows. An individual ("you" in the example of Figure 1) is connected with several other nodes in a network and is facing the one-shot choice problem to change at most one link: her options are to delete one of her existing links (a or d in the example), to create a link with one node that she is currently not directly connected to (b or c in the example), or not to change anything. Her payoff entirely depends on the network structure after completion of her choice.

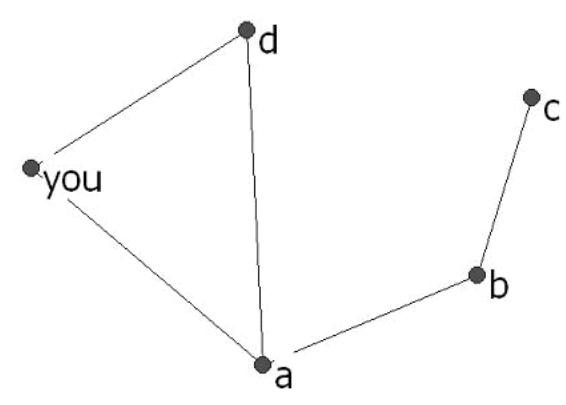

Figure 1: Example

\subsection{Prior Utility Models of Network Formation Decisions}

Economic theory traditionally models the utility that individual $i$ derives from choosing alternative $j$ as:

$$
U_{j}^{i}=f^{i}\left(\text { Payoff }_{j}^{i}\right)
$$

where Payoff ${ }_{j}^{i}$ is the payoff experienced by individual $i$ when she chooses alternative $j$ and $f^{i}$ is a strictly increasing function. 
Social preferences theory (for an overview see Fehr and Schmidt 2003) augments this model by allowing for the fact that in addition to their own payoff, individuals may take the payoff for other individuals into account when making their decisions. In this case, the utility that individual $i$ derives from choosing alternative $j$ is given by:

$$
U_{j}^{i}=f^{i}\left(\text { OwnPayoff }_{j}^{i},\left(\text { OthersPayoff }(q)_{j}^{i}\right)_{q \neq i}\right),
$$

where OwnPayoff ${ }_{j}^{i}$ is the payoff personally experienced by individual $i$ when she chooses alternative $j$, OthersPayoff $(q)_{j}^{i}$ is the payoff experienced by another individual $q$ when individual $i$ chooses alternative $j$, and $f^{i}($.$) is a function reflecting how individual i$ holds others-oriented payoff components in mind (e.g., inequity aversion, efficiency preferences, etc.).

\subsection{Hypotheses}

Our claim is that these prior utility models are not sufficient in explaining link choice behavior in the presence of network complexity. As humans have bounded rationality (Loasby 1976, Simon 1979, Camerer 1998), they cope with complexity in decision making by using simplifying heuristics, which commonly involve assessing a judgment object (e.g., linking choice alternative) using only the subset of properties of the object that are most accessible, i.e., that come most readily to mind, rather than using all relevant properties (Gilovich et al. 2002, Kahneman 2003, Gigerenzer et al. 1999). For example, when faced with a choice that is hard to resolve, people often search for a compelling rationale for choosing one alternative over another (Shafir et al. 1993). This is clearly illustrated in the literature about the effects of task complexity in several other contexts, like audit judgment (e.g., Bonner 1994) and consumer choice (e.g., Swait and Adamowicz 2001), but no research to date has addressed simplification rules that individuals use in making complex network formation decisions.

We propose two main types of simplifying heuristics that individuals use in making linking decisions: (i) they employ descriptive properties of the choice alternatives besides their normative payoff properties (Section 2.2.1) and (ii) they focus on only the most easily accessible parts of the normative payoff properties (Section 2.2.2). Furthermore, we examine whether both forms of simplification are stronger under more complex network decision making conditions (Section 2.2.3). 


\subsubsection{Descriptive Traits}

In the network formation setting the decision maker's payoff depends on the network structure after completion of her choice, where having more connections is on the one hand beneficial, since they provide access to additional resources, and on the other hand costly, for it takes time and effort to maintain them. It is typically a complex task for individuals to judge the exact payoff of link choice alternatives and we examine whether individuals use simplifying heuristics. A similar psychological process of judgment simplification is encountered in the literature about Conjunctive Probability Assessment, which shows that individuals make predictions based on a correlation they assume to exist between the assessment variable and some other variable (e.g., Broniarczyk \& Alba 1994).

Accordingly, we investigate whether individuals partly substitute the normative payoff value of a link choice alternative by descriptive attributes that can be determined more easily and that are qualitatively related to it. In particular, individuals can relatively easily see that there is a tradeoff between benefits and costs of more connections. Consequently, we hypothesize that they use as heuristic attributes (i) whether a link choice alternative involves link deletion or creation, and (ii) whether it concerns an isolated node, i.e., a node without direct links, or a central node, i.e., a node with three or more direct links. This is in line with Qualitative Process Theory, which suggests that human reasoning is more likely to depend on qualitative rather than quantitative relations (Forbus 1993). Therefore, it is examined whether individuals use the type of action or node as behavioral cues rather than the expected payoff.

Summarizing, we can formulate the following hypothesis:

Hypothesis 1 (effects of descriptive traits on link choice) An individual's network formation choices are affected by descriptive traits of link choice alternatives, i.e., whether an alternative involves link deletion (-) or creation (+), and whether it concerns an isolated node (+) or a central node (-).

Pursuing this line of reasoning, we formulate the utility that individual $i$ derives from choosing alternative $j$ with the following behavioral cues:

$$
U_{j}^{i}=f^{i}\left(\text { Payoff }_{j}^{i}, \text { Delete }_{j}, \text { Create }_{j}, \text { Central }_{j}, \text { Isolated }_{j}\right),
$$

where Payoff ${ }_{j}^{i}$ is the payoff experienced by individual $i$ when she chooses alternative $j$, and Delete ${ }_{j}$, Create $_{j}$, Central $_{j}$, and Isolated $_{j}$ are dummy variables indicating by 1 that alternative $j$ involves link deletion, link creation, a link with a central node, and a link with an isolated node, respectively. The 
function $f^{i}$ decreases in Delete ${ }_{j}$ and Central $_{j}$, and increases in Create ${ }_{j}$ and Isolated $_{j}$.

\subsubsection{Shifts in Payoff Traits}

Direct versus Indirect Payoff An important aspect of complexity in a network setting is that there can be value transferability, implying that an individual derives value not only from her direct neighbors, but also from her neighbors' neighbors, etc. (e.g., Bala and Goyal 2000, Jackson and Wolinsky 1996). This makes it more complex for individuals to judge the exact value of link choice alternatives, because besides evaluating the direct payoff of each link, they also have to be forwardlooking over indirect links.

Our hypothesis is that individuals tend to simplify by focusing most strongly on the payoff aspect that can be determined most easily: the value that is derived from direct neighbors only. The tendency to discount items in the utility function that are relatively more distant is known from research about time discounting (e.g., Frederick et al. 2002). Closely related to our hypothesis of networkshortsightedness are findings of spatial discounting, i.e., weighting spatially close features more strongly in the utility function than spatially distant features (e.g., Hannon 1994, Levinthal and March 1993).

Therefore, we hypothesize:

Hypothesis 2 (effects of indirect payoff on link choice) An individual's network formation choices are affected less strongly by payoff she derives from indirect nodes $(+)$ than by payoff from direct nodes $(+)$.

We capture this second proposed behavioral effect as follows in the utility that individual $i$ derives from choosing alternative $j$ :

$$
U_{j}^{i}=f^{i}\left(\text { DirectPayoff }_{j}^{i}, \text { IndirectPayoff }_{j}^{i}, \text { Delete }_{j}, \text { Create }_{j}, \text { Central }_{j}, \text { Isolated }_{j}\right),
$$

where DirectPayoff ${ }_{j}^{i}$ is the payoff experienced by individual $i$ from her direct neighbors when she chooses alternative $j$, IndirectPayoff ${ }_{j}^{i}=$ Payoff $_{j}^{i}-$ DirectPayoff $_{j}^{i}$, and $f^{i}$ increases in IndirectPayoff ${ }_{j}^{i}$ but more so in DirectPayoffi ${ }_{j}^{i}$.

Own versus Others' Payoff Social tradeoff is a further complicating factor in the network setting, implying that an individual's choices not only affect her own value, but also her neighbors' (and her neighbors' neighbors, etc.) (e.g., Bala and Goyal 2000, Jackson and Wolinsky 1996). This aspect of 
network formation choices makes it more complex for individuals with social preferences to judge the exact value of link choice alternatives, because besides their own payoff they also have to consider the payoff of (possibly many) other individuals.

Similarly to our investigation with respect to how individuals simplify the complexity of having to deal with indirect payoff, we also investigate whether individuals deal with the complexity of social tradeoff by focusing on the payoff aspect that can be determined most easily. In this case we examine whether individuals tend to focus more strongly on their own payoff and will pay relatively less attention to others' payoff due to the greater complexity of evaluating this social payoff. In the past, behavioral economists have found empirical evidence for social preferences, but mainly in lab environments where choice complexity was largely mitigated by explicit payoff information, which presents participants with the numerical payoff consequences of their choice options. However, Charness et al. (2004) have shown that individuals' social choices are affected by the variation of complexity resulting from such a comprehensive payoff overview.

Thus, we propose the following hypothesis:

Hypothesis 3 (effects of others' payoff on link choice) An individual's network formation choices are affected less strongly by their payoff consequences for other individuals $(+)$ when she is provided with a complete description of how the payoffs depend on these choices than when she is provided with a numerical payoff overview (+).

We include this third behavioral effect in the utility that individual $i$ derives from choosing alternative $j$ as follows:

$$
\begin{aligned}
& U_{j}^{i}=f^{i}\left(\text { OwnDirectPayoff }_{j}^{i} \text {, OwnIndirectPayoff }{ }_{j}^{i},\right. \\
& \text { (OthersDirectPayoff } \left.(q)_{j}^{i}\right)_{q \neq i},\left(\text { OthersIndirectPayoff }(q)_{j}^{i}\right)_{q \neq i}, \\
& \text { Delete } \left._{j}, \text { Create }_{j}, \text { Central }_{j}, \text { Isolated }_{j}\right) \text {, }
\end{aligned}
$$

where OwnDirectPayoff ${ }_{j}^{i}$ is the payoff personally experienced by individual $i$ from her direct neighbors when she chooses alternative $j$, OwnIndirectPayoff ${ }_{j}^{i}$ is the payoff personally experienced from indirect nodes, OthersDirectPayoff $(q)_{j}^{i}$ is the payoff experienced by another individual $q$ from her direct neighbors when individual $i$ chooses alternative $j$, OthersIndirectPayoff $(q)_{j}^{i}$ is the payoff experienced by another individual from indirect nodes, and $f^{i}$ is a function less increasing in (OthersPayoff $\left.(q)_{j}^{i}\right)_{q \neq i}$ when individual $i$ does not receive an explicit payoff overview than when she does. 


\subsubsection{Complexity Conditions}

Finally, we hypothesize that the different complexity aspects of networks may strengthen individuals' tendency to rely on the proposed heuristics. We include these moderating effects of complexity in the utility that individual $i$ derives from choosing alternative $j$ as follows:

$$
\begin{aligned}
U_{j}^{i}= & f^{i}\left(\text { OwnDirectPayoff }_{j}^{i}, \text { OwnIndirectPayoff }_{j}^{i},\right. \\
& \left(\text { OthersDirectPayoff }_{q}()_{j}^{i}\right)_{q \neq i},\left(\text { OthersIndirectPayoff }_{q}(q)_{j}^{i}\right)_{q \neq i}, \\
& \text { Delete }_{j}, \text { Create }_{j}, \text { Central }_{j}, \text { Isolated }_{j}, \\
& \text { Complexity }^{i} \times\left(\operatorname{OwnDirectPayoff}_{j}^{i}, \text { OwnIndirectPayoff }_{j}^{i},\right. \\
& \left.\left(\text { OthersDirectPayoff }(q)_{j}^{i}\right)_{q \neq i},\left(\operatorname{OthersIndirectPayoff}_{(q}\right)_{j}^{i}\right)_{q \neq i}, \\
& \text { Delete } \left.\left._{j}, \text { Create }_{j}, \operatorname{Central}_{j}, \text { Isolated }_{j}\right)\right),
\end{aligned}
$$

where Complexity ${ }^{i}$ is the network choice complexity that individual $i$ is facing and $f^{i}$ is a function in which the interaction effects with Complexity ${ }^{i}$ are included.

Structural Complexity First, we examine whether structural complexity in the network formation context affects decision making in a similar way as task complexity in the consumer choice context (e.g., Johnson and Payne 1985). More specifically, we investigate whether changes in structural features like increases in the number of nodes (and thus choice alternatives) in the network, will strengthen individuals' use of heuristics in terms of more strongly focusing on the role of descriptive traits in the utility function and relying more strongly on payoff function simplifications. In our analysis we focus on the feature connected position (i.e., the decision maker is linked to the network herself, like "you" in the example of Figure 1) as an important aspect of structural complexity, which makes it more difficult for the individual to assess the consequences of changes in the status quo and thus link choice alternatives. This brings us to the following hypotheses:

\section{Hypothesis 4 (moderating effects of structural complexity)}

Hypothesis 4.1 Structural complexity increases the impact of the descriptive traits of link choice alternatives (i.e., whether an alternative involves link deletion or creation and whether it concerns an isolated node or a central node) on link choice.

Hypothesis 4.2 Structural complexity decreases the impact of indirect payoff on link choice. 
Hypothesis 4.3 Structural complexity decreases the impact of others' payoff on link choice.

Value Transferability Another network feature regarded here is value transferability, which refers to the fact that an individual derives value not only from her direct neighbors, but also indirectly from her neighbors' neighbors, etc. (cf. Section 2.2.2). This network property makes it more complex for individuals to judge the exact value of link choice alternatives, because they have to be forwardlooking over indirect links. Value transferability therefore not only enables the heuristic of focusing on the part of the normative value that concerns payoff derived from direct neighbors, but furthermore stimulates the use of the other heuristics. This leads to the following hypotheses:

\section{Hypothesis 5 (moderating effects of value transferability)}

Hypothesis 5.1 Value transferability increases the impact of the descriptive traits of link choice alternatives (i.e., whether an alternative involves link deletion or creation and whether it concerns an isolated node or a central node) on link choice.

Hypothesis 5.2 Value transferability decreases the impact of others' payoff on link choice.

Social Tradeoff As a final complexity property, we consider social tradeoff, implying that an individual's choices not only affect her own value, but also her neighbors' (and her neighbors' neighbors, etc.) (cf. Section 2.2.2). This network property makes it more complex for individuals with social preferences to judge the exact value of link choice alternatives, because besides their own payoff they have to consider the payoff of (possibly many) other individuals. Therefore, social tradeoff not only enables the heuristic of focusing on the part of the normative value that concerns own payoff, but also has a strengthening effect on the role of the other heuristics. This can be formulated in the following hypotheses:

\section{Hypothesis 6 (moderating effects of social tradeoff)}

Hypothesis 6.1 Social tradeoff increases the impact of the descriptive traits of link choice alternatives (i.e., whether an alternative involves link deletion or creation and whether it concerns an isolated node or a central node) on link choice.

Hypothesis 6.2 Social tradeoff decreases the impact of indirect payoff on link choice.

Our hypotheses are illustrated in Figure 2. 


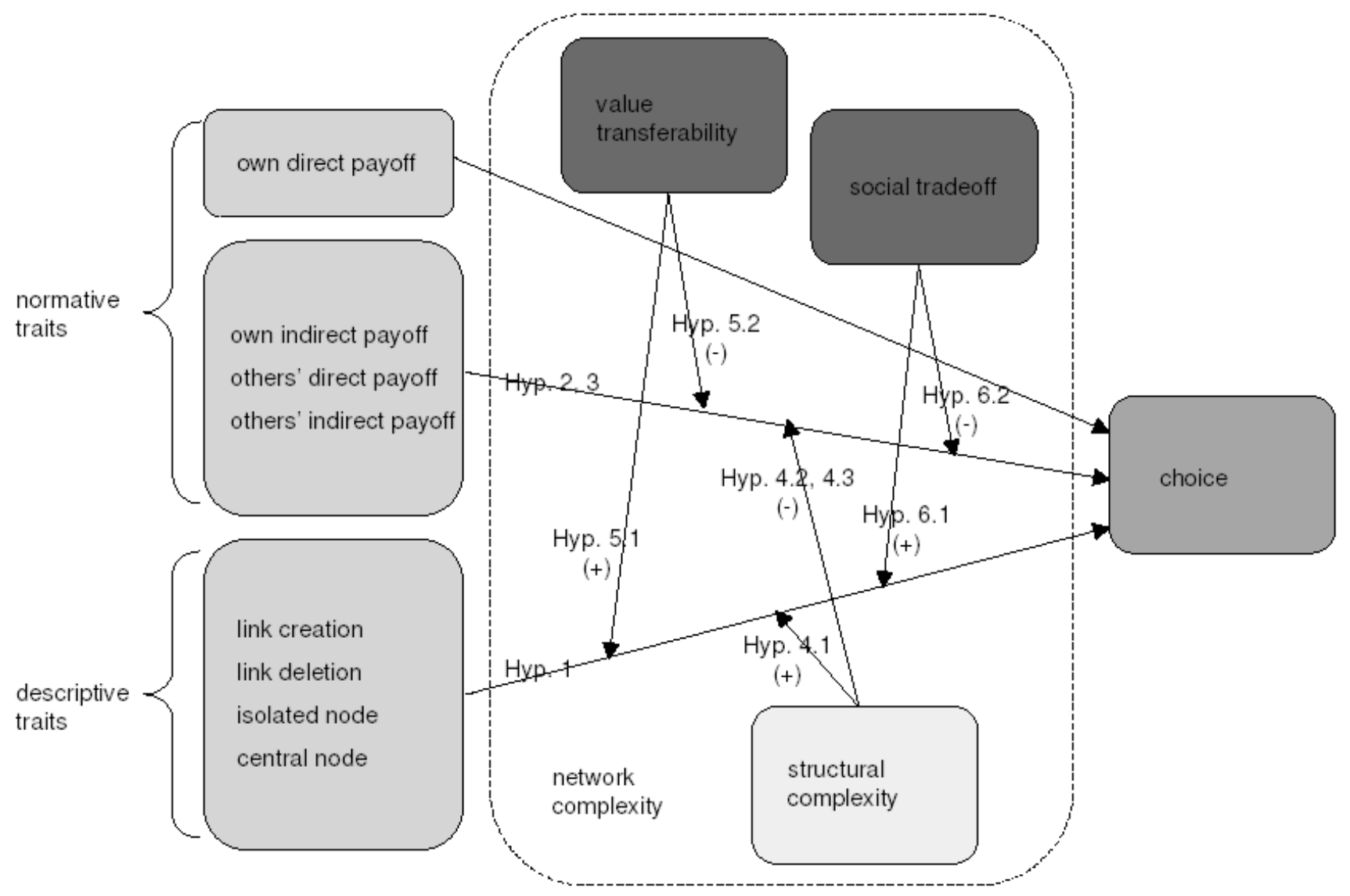

Figure 2: Hypothesized Model

\section{Methods}

In this section we describe the experimental design as well as the parametric approach used for testing our hypotheses.

\subsection{Experimental Design}

Our experiment presented participants with six choice problems similar to that in Figure 1. In these problems a participant was allowed to change at most one direct link, i.e., to delete a link that already exists between her and another node, to create a link between her and another node if there is not yet one, or to change nothing. The choice problems are illustrated in Tables 7 and 8, Appendix B.

To test for the hypothesized shifts in heuristic behavior due to value transferability (equation (2) versus equation (1)) and social tradeoff (equation (3) versus equation (1)), we employed four experimental treatments where these two characteristics were the between-subjects factors. Thus, each participant faced one of four particular complexity conditions (see Section 2.2.3). The experimental 
design is summarized by Table 1. A within-subject manipulation for the treatments social and both will be introduced later in this section.

\begin{tabular}{|c|c|c|c|}
\hline \multirow{4}{*}{$\begin{array}{l}\text { value } \\
\text { transferability? }\end{array}$} & & \multicolumn{2}{|c|}{ social tradeoff? } \\
\hline & \multirow{3}{*}{$\begin{array}{l}\text { NO } \\
\text { YES }\end{array}$} & $\mathrm{NO}$ & YES \\
\hline & & none & social (part 1, part 2) \\
\hline & & transfer & both (part 1, part 2) \\
\hline
\end{tabular}

Table 1: Experimental Design

Each participant was confronted with a payoff function matching her condition. This function reflects the benefits and costs of link formation and was explained in words to the participants in the instructions (see Appendix A). For a participant $i$ in between-subject treatments none and social there is no value transferability, so value is derived from direct neighbors only. The payoff function is then given by:

$$
\Pi_{i}=\left\{\begin{array}{ccc}
\sum_{j \in N_{i}} \frac{1}{\mu_{i} \cdot \mu_{j}} & \text { if } & \mu_{i}>0 \\
0 & \text { if } & \mu_{i}=0
\end{array}\right.
$$

where $N_{i}$ is the set of nodes with whom $i$ has a direct link, node $j$ is a neighbor of $i$ if $j \in N_{i}$, and $\mu_{i}=\left|N_{i}\right|$ is the number of neighbors of $i$, the degree of $i$.

For between-subject treatments transfer and both there is value transferability, so value is derived from direct as well as indirectly connected nodes. The payoff function is then given by:

$$
\Pi_{i}=\left\{\begin{array}{ccc}
\sum_{j \in \bar{N}_{i}} \sum_{p \in \mathcal{P}_{i, j}} \frac{1}{\mu_{i} \cdot \mu_{j} \cdot \prod_{k \in \breve{P}}\left(\mu_{k}\right)^{2}} & \text { if } \quad \mu_{i}>0 \\
0 & \text { if } \quad \mu_{i}=0
\end{array}\right.
$$

where $\bar{N}_{i}$ is the set of nodes with whom $i$ has either a direct or an indirect link, $\mathcal{P}_{i, j}$ is the set of paths between $i$ and $j$, where a path is defined as a sequence of consecutive edges without repeated nodes, $\breve{P}$ is the set of nodes on path $p$ between $i$ and $j$ excluding $i$ and $j$ themselves, and $\mu_{i}$ is the degree of $i$. In the instructions, these payoff functions are not presented in formulas but in easy verbal descriptions, illustrated by an example (see Appendix A).

For between-subject treatments none and transfer there is no social tradeoff. The participants were informed that nobody else was affected by their choices. For treatments social and both there is social tradeoff. The participants were informed that the payoffs for the other nodes in the choice problems are determined analogously to their own payoff, and that the total payoffs created for these other nodes due to their choices are divided equally among the other participants in the room. Thus, 
a simple form of social preferences, not regarding distributional issues, was evoked. No information or feedback about the tasks and choices of the other participants was provided during the experiment in order to rule out strategic motivations.

Three of the six problems involves a connected position and the other half an isolated position for the participant (see Tables 7 and 8, Appendix B). Thus, the structural complexity characteristic connected position was included as within-subject factor in the experimental design. The number of nodes was kept constant in all six choice problems. Pilot studies conducted by the authors before the experiment indicated that several other structural complexity features like the exact number of links and the number of visual crossings between links do not affect participants' choices. Still, to avoid biases due to such other structural factors, the order of choice problems was rotated among participants.

To control for individual differences, particularly in social preferences, for participants in betweensubject treatments social and both where payoff for other participants had to be considered, an additional part was added to experiment. This was exactly the same as the first part, but for each choice option the payoff for the participant as well as for the others were mentioned explicitly. This is illustrated in Figure 3, Appendix B. Charness, Frechette, and Kagel (2004) showed that people behave differently in gift-exchange games when they receive a comprehensive payoff table, and the objective of this additional manipulation is to test in how far participants take others' payoff into account when the complexity of doing so is removed. The payoffs for all choice problems are given in Table 9 in the same appendix. Thus, for the between-subject treatments social and both, payoff information was also incorporated as a within-subjects factor.

The experiment took place in a computer lab with students and employees of various faculties of Maastricht University, the Netherlands. In each of the four between-subject conditions there were 28 participants in batches of 7 . After each choice, feedback was given to the participant about the payoff she earned for herself and if relevant for the other participants in the room, and the maximum and minimum number of points that could have been earned in the specific choice problem. Participants were informed how the payoffs they earn in the experiment are converted into cash euros afterwards, see Sections 6.1 and 6.2, Appendix A for details. They could only start the experiment after answering control questions correctly and after two really paid-out practice rounds with only three choice alternatives, see Table 6, Appendix B. At the end they were asked to comment on the way they made their choices in a debriefing part. 


\subsection{Mixed Logit Estimations}

We perform a comprehensive parametric test of our hypotheses by estimating a mixed (i.e., random parameters) logit model (Hensher et al. 2005). This enables us to establish the roles of several attributes of link alternatives in the network formation process, while allowing for heterogeneity across individuals. We assume that (without numerical payoff information), the utility that individual $i$ derives from choosing alternative $j$ in choice problem $t$ is given by:

$$
\begin{aligned}
U_{t j}^{i} & =\sum_{k \in K} \beta_{k}^{i} \cdot P_{k t j}^{i}+\sum_{k \in K} \gamma_{k}^{i} \cdot D_{k t j}+\sum_{k \in K} \zeta_{k} \cdot C_{t} \cdot P_{k t j}^{i}+\sum_{k \in\{2,3\}} \eta_{k} \cdot C_{t} \cdot D_{k t j} \\
& +\sum_{k \in\{1,2\}} \theta_{k} \cdot S^{i} \cdot P_{k t j}^{i}+\sum_{k \in K} \xi_{k} \cdot S^{i} \cdot D_{k t j}+\sum_{k \in\{1,3\}} \varphi_{k} \cdot T^{i} \cdot P_{k t j}^{i}+\sum_{k \in K} \chi_{k} \cdot T^{i} \cdot D_{k t j}+\varepsilon_{t j}^{i},
\end{aligned}
$$

where:

$K \quad$ is the set of indices $\{1,2,3,4\}$,

$P_{1 t j}^{i} \quad$ is the own, direct payoff generated for an individual $i$

when in choice problem $t$ she chooses alternative $j$,

$P_{2 t j}^{i} \quad$ is the own, indirect payoff,

$P_{3 t j}^{i} \quad$ is the direct payoff generated for the other participants,

$P_{4 t j}^{i} \quad$ is the indirect payoff generated for the other participants,

$D_{1 t j}$ is a dummy variable indicating link deletion,

$D_{2 t j}$ is a dummy variable indicating link creation,

$D_{3 t j}$ is a dummy variable indicating a central node, i.e., with three or more direct links,

$D_{4 t j}$ is a dummy variable indicating an isolated node, i.e., without direct links,

$C_{t} \quad$ is a dummy variable indicating a connected start position, i.e., not isolated,

$S^{i} \quad$ is a dummy variable indicating social tradeoff, i.e., treatment social or both,

$T^{i} \quad$ is a dummy variable indicating value transferability, i.e., treatment transfer or both, and

$\varepsilon_{t j}^{i} \quad$ is a stochastic variable drawn from a standard Gumble distribution.

In the random parameter $\beta_{1}^{i}$, superscript $i$ allows for heterogeneity due to individuals' personal preferences as follows:

$$
\beta_{1}^{i}=\beta_{1}+\nu_{1}^{i},
$$

where $\nu_{1}^{i}$ is a stochastic variable drawn from a normal distribution. Analogously, random parameters 
are included for the other main effects of the payoff and descriptive traits on choice, but in quest of parsimony not for the interaction effects. Note that in the variables, superscript $i$ allows for heterogeneity due to the between-subject treatment individuals participate in (e.g., in none and social participants do not receive any indirect payoffs and in none and transfer participants do not generate any payoffs for the other participants in the room). Furthermore, notice that interactions between $C$ and $D_{1}, C$ and $D_{4}, S$ and $P_{3}, S$ and $P_{4}, T$ and $P_{2}$, or $T$ and $P_{4}$ do not provide additional information to $D_{1}, D_{4}, P_{3}, P_{4}, P_{2}$, or $P_{4}$, respectively, and therefore are not included.

Then, under the usual assumptions, the unconditional probability that individual $i$ will choose alternative $j$ equals the expected value of the logit probability over all possible values of the random parameters. The model is estimated by Maximum Likelihood with NLOGIT 3.0, Econometric Software, Inc., implementing 500 Halton draws in the Monte Carlo simulation.

Finally, to investigate the impact of providing participants with an explicit payoff overview, which should remove the complexity effects, a similar model is estimated for the data with and without numerical payoff information:

$$
\begin{aligned}
U_{t j}^{i} & =\sum_{k \in K} \beta_{k}^{i} \cdot P_{k t j}^{i}+\sum_{k \in K} \gamma_{k}^{i} \cdot D_{k t j}+\sum_{k \in K} \zeta_{k} \cdot C_{t} \cdot P_{k t j}^{i}+\sum_{k \in\{2,3\}} \eta_{k} \cdot C_{t} \cdot D_{k t j} \\
& +\sum_{k \in\{1,2\}} \theta_{k} \cdot S^{i} \cdot P_{k t j}^{i}+\sum_{k \in K} \xi_{k} \cdot S^{i} \cdot D_{k t j}+\sum_{k \in\{1,3\}} \varphi_{k} \cdot T^{i} \cdot P_{k t j}^{i}+\sum_{k \in K} \chi_{k} \cdot T^{i} \cdot D_{k t j} \\
& +\sum_{k \in K} \psi_{1 k} \cdot I_{t}^{i} \cdot P_{k t j}^{i}+\sum_{k \in K} \psi_{2 k} \cdot I_{t}^{i} \cdot D_{k t j}+\sum_{k \in K} \psi_{3 k} \cdot I_{t}^{i} \cdot C_{t} \cdot P_{k t j}^{i} \\
& +\sum_{k \in\{2,3\}} \psi_{4 k} \cdot I_{t}^{i} \cdot C_{t} \cdot D_{k t j}+\sum_{k \in\{1,3\}} \psi_{5 k} \cdot I_{t}^{i} \cdot T^{i} \cdot P_{k t j}^{i}+\sum_{k \in K} \psi_{6 k} \cdot I_{t}^{i} \cdot T^{i} \cdot D_{k t j}+\varepsilon_{t j}^{i},
\end{aligned}
$$

where $I_{t}^{i}$ is a dummy variable indicating by 1 that participant $i$ was provided with payoff information when solving choice problem $t$. Note that interactions between $I$ and $S \cdot P$ or $I$ and $S \cdot D$ do not provide additional information to $S \cdot P$ or $S \cdot D$, respectively, and therefore are not included.

\section{Results}

\subsection{Some Illustrative Descriptive Results}

Before turning to a more formal analysis, we first present some illustrative results for the choices made in the different conditions. Hereby the focus is on choice problem 5 (Table 2).

The use of descriptive traits as proposed by Hypothesis 1 can best be illustrated by the results under complexity condition social (second row Table 2). Although changing nothing is the status quo, 


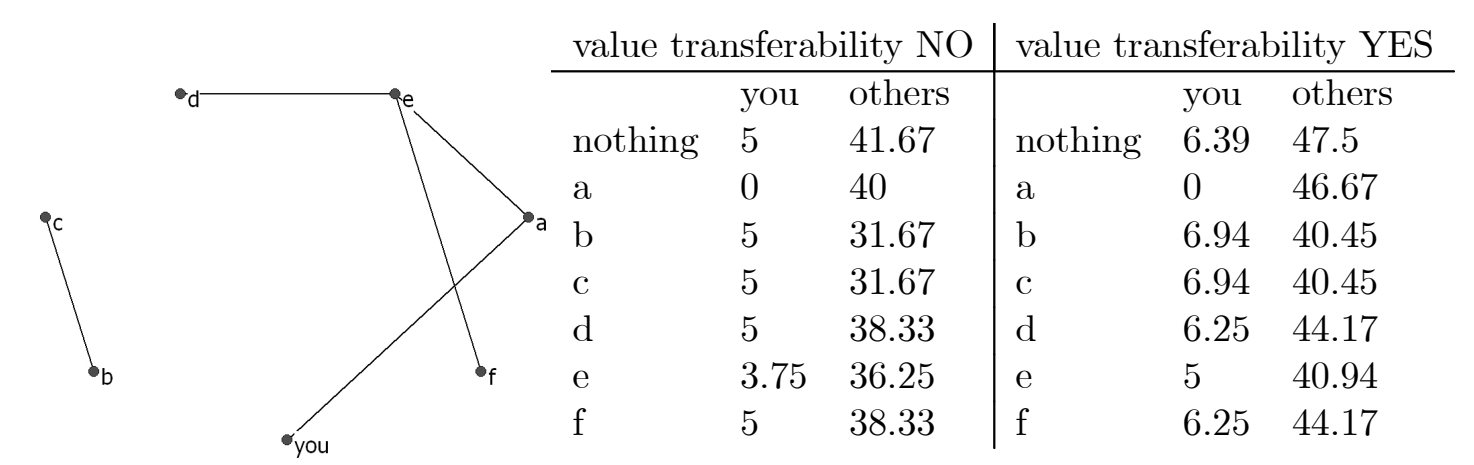

\begin{tabular}{|c|c|}
\hline none / payoff info NO & transfer / payoff info NO \\
\hline choice $\quad \%$ & choice $\quad \%$ \\
\hline nothing $\quad 43.3$ & nothing $\quad 17.9$ \\
\hline 0.0 & 0.0 \\
\hline 40.0 & 60.7 \\
\hline 10.0 & 7.1 \\
\hline 3.3 & 0.0 \\
\hline 0.0 & 3.6 \\
\hline 3.3 & 10.7 \\
\hline social / payoff info NO & social / payoff info YES \\
\hline choice $\quad \%$ & choice \\
\hline nothing 10.7 & nothing $\quad 53.6$ \\
\hline 0.0 & 0.0 \\
\hline 39.3 & 21.4 \\
\hline 7.1 & 7.1 \\
\hline 17.9 & 7.1 \\
\hline 0.0 & 3.6 \\
\hline 25.0 & 7.1 \\
\hline both / payoff info NO & both / payoff info YES \\
\hline choice $\quad \%$ & choice $\quad \%$ \\
\hline nothing $\quad 35.7$ & nothing 25.0 \\
\hline 0.0 & 0.0 \\
\hline 28.6 & 46.4 \\
\hline 14.3 & 25.0 \\
\hline 7.1 & 3.6 \\
\hline 7.1 & 0.0 \\
\hline 7.1 & 0.0 \\
\hline
\end{tabular}

Table 2: Descriptive Results Choice Problem 5

the first option in the list, and the single option that is both normatively optimal for themselves and for the others, only $10.7 \%$ of the respective participants opts for this alternative. However, in the second part of the experiment, when payoff information is given, thus eliminating network complexity, this proportion grows to $53.6 \%$. This corresponds to the use of the descriptive trait link creation as a choice heuristic, where creating a link is qualitatively associated with higher payoff.

In complexity condition none (first row Table 2), without value transferability and social tradeoff, all participants choose one of the normatively optimal alternatives, i.e., nothing, b, c, d, or f. However, 
in condition transfer, where value transferability is included, only $67.8 \%$ of the respective participants choose one of the normatively optimal alternatives, i.e., b or c, whereas $96.4 \%$ of the choices would be optimal if only direct payoff is considered. Although causality is undecided, this pattern is in line with Hypothesis 2.

In complexity condition social (second row Table 2), where social tradeoff is included, only $10.7 \%$ of the respective participants turns out to opt for the option not to change anything, which reveals social preferences in the sense that it maximizes the payoffs for the other participants, given maximum own payoff. All these participants still maximize their own payoff though. However, in the second part of the experiment, when payoff information is given, thus eliminating network complexity, $53.6 \%$ of the same participants prefers this option. This pattern corresponds with Hypothesis 3.

In complexity condition both (third row Table 2), with both value transferability and social tradeoff, only $42.9 \%$ of the respective participants chooses one of the options with optimal own payoff, i.e., b or c, whereas the rest seems to reveal social preferences in the sense that they reduce their own payoff in order to increase others' payoffs. Note that $21.3 \%$ even chooses a Pareto inferior option, i.e., a, d, e, or f, and $92.9 \%$ still maximizes their own, direct payoff. However, in the second part of the experiment, when payoff information is given, thus eliminating network complexity, the proportion with optimal own payoff increases to $71.4 \%$. Also, only $3.6 \%$ chooses a Pareto inferior option. These findings further corroborate Hypotheses 2 and 3.

Further descriptive results, primarily from the debriefing part, are given in Appendix C.

\subsection{Mixed Logit Results}

A comprehensive parametric test of the hypotheses is conducted by estimating a mixed logit model across all experimental conditions for the first part of the experiment without numerical payoff information (Section 4.2.1) and a similar model including interaction effects of such payoff information (Section 4.2.2). A p-value of 0.05 is taken as cut-off value for significance.

\subsubsection{With Description of Choice Consequences}

Table 3 summarizes the estimation results for the first part of the experiment in which participants were asked to make network formation decisions based on a complete description of how the payoffs depend on these choices and no numerical payoff overview was provided.

We find support for the use of descriptive traits additional to the normative payoff in making decisions (Hypothesis 1): link deletion and central node have a significant negative effect on choice and 


\begin{tabular}{|c|c|c|c|}
\hline variable & parameter & $\begin{array}{r}\text { estimated mean } \\
(\mathrm{p} \text {-value })\end{array}$ & $\begin{array}{l}\text { estimated standard } \\
\text { deviation ( } \mathrm{p} \text {-value) }\end{array}$ \\
\hline own direct payoff & $\beta_{1}^{i}$ & $.33(.00)$ & $.00(1.0)$ \\
\hline own indirect payoff & $\beta_{2}^{i}$ & $-.16(.53)$ & $.02(.96)$ \\
\hline others' direct payoff & $\beta_{3}^{i}$ & $.02(.68)$ & $.00(.99)$ \\
\hline others' indirect payoff & $\beta_{4}^{i}$ & $-.08(.57)$ & $.00(.98)$ \\
\hline link deletion & $\gamma_{1}^{i}$ & $-1.01(.00)$ & $.00(1.0)$ \\
\hline link creation & $\gamma_{2}^{i}$ & $3.10(.00)$ & $1.79(.00)$ \\
\hline central node & $\gamma_{3}^{i}$ & $-2.30(.00)$ & $1.34(.00)$ \\
\hline isolated node & $\gamma_{4}^{i}$ & $2.65(.00)$ & $.01(.99)$ \\
\hline connected ${ }^{*}$ own direct payoff & $\zeta_{1}$ & $-.43(.00)$ & \\
\hline connected ${ }^{*}$ own indirect payoff & $\zeta_{2}$ & $1.12(.00)$ & \\
\hline connected $*$ others' direct payoff & $\zeta_{3}$ & $.02(.77)$ & \\
\hline connected ${ }^{*}$ others' indirect payoff & $\zeta_{4}$ & $.35(.06)$ & \\
\hline connected $*$ link creation & $\eta_{2}$ & $-3.16(.00)$ & \\
\hline connected $*$ central node & $\eta_{3}$ & $-2.36(.00)$ & \\
\hline social tradeoff $*$ own direct payoff & $\theta_{1}$ & $-.05(.56)$ & \\
\hline social tradeoff $*$ own indirect payoff & $\theta_{2}$ & $.72(.03)$ & \\
\hline social tradeoff $*$ link deletion & $\xi_{1}$ & $-.19(.66)$ & \\
\hline social tradeoff $*$ link creation & $\xi_{2}$ & $.64(.22)$ & \\
\hline social tradeoff $*$ central node & $\xi_{3}$ & $.36(.46)$ & \\
\hline social tradeoff $*$ isolated node & $\xi_{4}$ & $-.26(.62)$ & \\
\hline transferability ${ }^{*}$ own direct payoff & $\varphi_{1}$ & $-.20(.01)$ & \\
\hline transferability $*$ others' direct payoff & $\varphi_{3}$ & $.15(.02)$ & \\
\hline transferability $*$ link deletion & $\chi_{1}$ & $.31(.47)$ & \\
\hline transferability $*$ link creation & $\chi_{2}$ & $-.44(.39)$ & \\
\hline transferability $*$ central node & $\chi_{3}$ & $2.76(.00)$ & \\
\hline transferability $*$ isolated node & $\chi_{4}$ & $-1.17(.03)$ & \\
\hline
\end{tabular}

Table 3: Mixed Logit Estimations Without Payoff Information

link creation and isolated node a significant positive effect, implying that compared to doing nothing, individuals have an aversion for deleting links and a preference for creating links, and compared to a node with one or two links, individuals prefer isolated choice alternatives and dislike nodes with more links, additional to the related payoff consequences.

Moreover, we find support for Hypothesis 2, since the indirect payoff does not significantly influence choice, and preliminary support for Hypothesis 3, since others' payoff does not significantly influence choice.

In case of a connected position for the decision maker and value transferability, the role of the own, direct payoff even significantly decreases, which is in line with Hypotheses 4.1 and 5.1 respectively. However, the complexity conditions do not simply strengthen the basic use of descriptive traits in all cases: under a connected position the tendency to link creation decreases and under value transferability respondents reverse their preference for isolated nodes and avoidance of central nodes. The 
data do not support Hypothesis 6.1 since under social tradeoff, there is no significant switch from normative to descriptive traits.

Surprisingly, in case of a connected position for the decision maker, there seems to be a switch of focus from direct to indirect payoff, which contradicts Hypothesis 4.2. The role of indirect payoff is also significantly larger in case of social tradeoff, which is the opposite interaction effect from what is predicted by Hypothesis 6.2 .

Hypothesis 4.3 is not supported by the data since there is no significant effect of any of the interaction terms of connected position and others' payoff. Hypothesis 5.2 is rejected by the data since the interaction term of value transferability and others' payoff is significantly positive.

Respondents' heuristic behavior significantly varies with respect to the preference for link creation and the avoidance of central nodes.

\subsubsection{With Numerical Payoff Information}

The estimation results for all experimental treatments including the interaction effects of a comprehensive payoff overview are given in Table 4.

With this model we find the same support for the use of descriptive traits (Hypotheses 1, 4.1, and 5.1) and the focus on own, direct payoff (Hypotheses 2 and 3) in the case without payoff information. Moreover, we again find the surprising effect of indirect payoff in case of structural complexity of a connected position for the decision maker and in case of social tradeoff, contradicting Hypotheses 4.2 and 6.2. Also the role of other participants' payoff is again significantly larger in case of value transferability, which is the opposite interaction effect from what is predicted by Hypothesis 5.2. Once more we do not find significant results for the effects predicted by Hypotheses 4.3 and 6.1. Lastly, we again find heterogeneity among participants with respect to their preference for link creation and avoidance of central nodes.

More importantly, with this model we find evidence for the role of payoff information indicating that substitution of the exact value of a link choice alternative by more accessible, related heuristic attributes (particular parts of normative traits as well as descriptive traits) is dependent on complexity. Especially, the significant positive effect of others' payoff under payoff information and a connected position for the decision maker confirms that without payoff information, participants are more inclined to be affected by the payoff consequences for other participants than actually reflected in their choices. This completes the proof for Hypothesis 3. 


\begin{tabular}{|c|c|c|c|}
\hline variable & par. & est. mean (p) & est. s.d. (p) \\
\hline own direct payoff & $\beta_{1}^{i}$ & $0.33(.00)$ & $0.00(.97)$ \\
\hline own indirect payoff & $\beta_{2}^{i}$ & $-0.14(.57)$ & $0.01(.98)$ \\
\hline others' direct payoff & $\beta_{3}^{i}$ & $0.02(.66)$ & $0.00(.97)$ \\
\hline others' indirect payoff & $\beta_{4}^{i}$ & $-0.08(.58)$ & $0.00(1.0)$ \\
\hline link deletion & $\gamma_{1}^{i}$ & $-1.00(.00)$ & $0.00(.99)$ \\
\hline link creation & $\gamma_{2}^{i}$ & $3.01(.00)$ & $1.73(.00)$ \\
\hline central node & $\gamma_{3}^{i}$ & $-2.39(.00)$ & $1.42(.00)$ \\
\hline isolated node & $\gamma_{4}^{i}$ & $2.61(.00)$ & $0.04(.93)$ \\
\hline connected ${ }^{*}$ own direct payoff & $\zeta_{1}$ & $-0.43(.00)$ & \\
\hline connected $*$ own indirect payoff & $\zeta_{2}$ & $1.11(.00)$ & \\
\hline connected ${ }^{*}$ others' direct payoff & $\zeta_{3}$ & $0.01(.84)$ & \\
\hline connected ${ }^{*}$ others' indirect payoff & $\zeta_{4}$ & $0.34(.07)$ & \\
\hline connected $*$ link creation & $\eta_{2}$ & $-3.02(.00)$ & \\
\hline connected $*$ central node & $\eta_{3}$ & $-2.36(.00)$ & \\
\hline social tradeoff $*$ own direct payoff & $\theta_{1}$ & $-0.04(.62)$ & \\
\hline social tradeoff $*$ own indirect payoff & $\theta_{2}$ & $0.66(.04)$ & \\
\hline social tradeoff $*$ link deletion & $\xi_{1}$ & $-0.19(.66)$ & \\
\hline social tradeoff $*$ link creation & $\xi_{2}$ & $0.60(.23)$ & \\
\hline social tradeoff $*$ central node & $\xi_{3}$ & $0.39(.44)$ & \\
\hline social tradeoff $*$ isolated node & $\xi_{4}$ & $-0.18(.74)$ & \\
\hline transferability ${ }^{*}$ own direct payoff & $\varphi_{1}$ & $-0.19(.01)$ & \\
\hline transferability $*$ others' direct payoff & $\varphi_{3}$ & $0.15(.02)$ & \\
\hline transferability $*$ link deletion & $\chi_{1}$ & $0.30(.48)$ & \\
\hline transferability $*$ link creation & $\chi_{2}$ & $-0.54(.28)$ & \\
\hline transferability $*$ central node & $\chi_{3}$ & $2.81(.00)$ & \\
\hline transferability $*$ isolated node & $\chi_{4}$ & $-1.10(.04)$ & \\
\hline payoff info $*$ own direct payoff & $\psi_{11}$ & $0.31(.04)$ & \\
\hline payoff info $*$ own indirect payoff & $\psi_{12}^{11}$ & $1.65(.03)$ & \\
\hline payoff info * others' direct payoff & $\psi_{13}$ & $0.01(.91)$ & \\
\hline payoff info $*$ others' indirect payoff & $\psi_{14}$ & $-0.03(.91)$ & \\
\hline payoff info $*$ link deletion & $\psi_{21}^{14}$ & $-1.00(.15)$ & \\
\hline payoff info $*$ link creation & $\psi_{22}$ & $0.20(.87)$ & \\
\hline payoff info ${ }^{*}$ central node & $\psi_{23}$ & $-1.21(.24)$ & \\
\hline payoff info $*$ isolated node & $\psi_{24}$ & $-1.54(.04)$ & \\
\hline payoff info $*$ connected $*$ own direct payoff & $\psi_{31}$ & $-0.41(.02)$ & \\
\hline payoff info $*$ connected $*$ own indirect payoff & $\psi_{32}$ & $-1.49(.05)$ & \\
\hline payoff info ${ }^{*}$ connected ${ }^{*}$ others' direct payoff & $\psi_{33}$ & $0.19(.04)$ & \\
\hline payoff info $*$ connected $*$ others' indirect payoff & $\psi_{34}$ & $0.72(.02)$ & \\
\hline payoff info $*$ connected $*$ link creation & $\psi_{42}$ & $-1.34(.30)$ & \\
\hline payoff info $*$ connected $*$ central node & $\psi_{43}$ & $3.12(.04)$ & \\
\hline payoff info $*$ transferability $*$ own direct payoff & $\psi_{51}$ & $-0.26(.23)$ & \\
\hline payoff info $*$ transferability $*$ others' direct payoff & $\psi_{53}$ & $0.10(.45)$ & \\
\hline payoff info $*$ transferability $*$ link deletion & $\psi_{61}$ & $-0.17(.87)$ & \\
\hline payoff info $*$ transferability $*$ link creation & $\psi_{62}$ & $3.19(.00)$ & \\
\hline payoff info $*$ transferability $*$ central node & $\psi_{63}$ & $-2.99(.03)$ & \\
\hline payoff info $*$ transferability $*$ isolated node & $\psi_{64}$ & $-1.11(.33)$ & \\
\hline
\end{tabular}

Table 4: Mixed Logit Estimations Including Payoff Information 


\subsubsection{Robustness}

In this section, we check whether our estimation results are robust for several control variables.

Learning Effects First of all, the main model for all between-subject treatments without payoff information is re-estimated including the control dummy variable experience, which tracks whether the respective participant at the moment of choice was solving either one of her last three problems or one of her first three, as an additional interaction. We find that the behavioral effects do not change and that the overall model fit does not improve, but the interactions of experience and some descriptive traits turn out to be significant. The respective signs of the parameter estimations are such that the use of these heuristics is stronger for more experienced individuals, so the behavioral effects in network formation decisions as explored in the current paper are not transitory.

Order Effects Next, the main model for all between-subject treatments without payoff information is re-estimated including the control dummy variable primacy, which tracks whether the respective alternative was listed either as one of the first four or as one of the last four choice options, as an additional choice predictor. Again we find that the behavioral effects remain and that the overall model fit hardly improves, whereas the respective parameter estimate is positive and significant.

Random Error Effects Finally, we compare our model to a simpler, more restricted model based on the prior predictions as described in Section 2.1 without our behavioral effects. In line with quantal response theory (e.g., McKelvey and Palfrey 1995, Charness and Jackson 2006), we add a random component to the individual decision strategy. The variance of this random component is allowed to vary between complexity conditions. Thus, we assume that without payoff information, the utility that individual $i$ derives from choosing alternative $j$ in choice problem $t$ is given by:

$$
U_{t j}^{i}=\beta_{1}^{i} \cdot\left(P_{1 t j}^{i}+P_{2 t j}^{i}\right)+\beta_{2}^{i} \cdot\left(P_{3 t j}^{i}+P_{4 t j}^{i}\right)+\varepsilon_{t j}^{i},
$$

where the random parameter $\beta_{1}^{i}$ is allowed to linearly depend on the complexity conditions $C_{t}, S^{i}$, and $T^{i}$. This rival model turns out to perform worse in terms of model fit (i.e., the Akaike Information Criterion increases from 3.47 to 3.84), strengthening our claim of systematic, heuristic effects of complexity on link choice behavior. 


\section{Discussion}

\section{$5.1 \quad$ Results}

This study shows that network complexity influences individual link choice behavior in a systematic way, since it affects individuals' use of heuristics in which the normative target attribute of a link choice alternative (i.e., payoff for themselves and possibly for other individuals) is assessed by substituting more accessible properties of that alternative. These heuristic attributes can either be parts of the normative traits: the payoff from their direct neighbors (Hypothesis 2) and their own payoff (Hypothesis 3), or descriptive traits: whether a link choice alternative involves link deletion or creation and whether it concerns an isolated or a central node (Hypothesis 1). Specifically, individuals tend to neglect indirect and others' payoff whereas they do significantly consider their own, direct payoff, they have an aversion for deleting links and a preference for creating links, and they prefer isolated choice alternatives and dislike nodes with more than two direct links, additional to the related payoff consequences. Furthermore, we find that complexity of one type can moderate individuals' dealing with a complex feature of another type. In particular, facing the structural complexity of a connected position (Hypothesis 4.1) or facing value transferability (Hypothesis 5.1), individuals tend to rely more strongly on descriptive traits. A summary of the results is provided in Table 5.

An explanation for the lack of support of the hypotheses related to social tradeoff is suggested by participants' replies to the debriefing questions. It seems that individuals consciously decide to completely neglect any payoff they generate for other people as soon as they are confronted with social tradeoff in a complex network setting. Therefore, a connected position and value transferability are not able to still strengthen their tendency to focus on own payoff (Hypotheses 4.3 and 5.2), and it is not necessary for them to also use other heuristics to deal with the added complexity of social tradeoff (Hypotheses 6.1 and 6.2).

\begin{tabular}{|l|l|}
\hline Hypothesis & Result \\
\hline behavioral effects on network formation & \\
1: use of descriptive traits & supported \\
2: focus on direct payoff & supported \\
3: focus on own payoff & supported \\
\hline moderating effects of network complexity & \\
4: structural complexity & partially supported \\
5: value transferability & partially supported \\
6: social tradeoff & not supported \\
\hline
\end{tabular}

Table 5: Summary Experimental Results 
The opposite effects for Hypotheses 4.2, 5.2, and 6.2 are more difficult to account for. It might be that individuals need a trigger to realize that it makes sense to consider payoff for others, namely that value is transferred over links (Hypothesis 5.2). Vice versa, the fact that their choices also influence other participants' payoff might be a trigger to make individuals realize that indirect payoff is also relevant (Hypothesis 6.2). With respect to Hypothesis 4.2, it might be that individuals need the feeling of safety from the zero payoff by being connected before being able to consider indirect payoff as to optimize their payoff.

The model estimations with inclusion of payoff information confirm that individuals' heuristic focus on the own, direct part of the normative trait and consideration of descriptive traits is based on the complexity of the network setting. This is also illustrated by the descriptive statistics presented in Section 4.1: the same individuals make significantly different decisions in the part of the experiment with simplifying payoff information than in the part without. In particular, this finishes the proof of Hypothesis 3, since apparently not all insignificant effects of payoff for others on choice are simply due to a lack of social preferences.

\subsection{Implications}

The current research pioneers the issue of behavioral effects in individual decisions of network formation. Our results should raise interest in future research into this realm, for they have important implications for experimental research as well as application areas of network formation.

For instance, experimental research practice is often disposed to make the payoff consequences of choices as transparent as possible for participants as to prevent biased findings due to their wrong understanding of the instructions. However, we claim that this modifies participants' behavior in a systematic way, since it eliminates complexity that they otherwise would handle by using heuristics. As we have shown in Section 4.2.3, these changes in behavior cannot accurately be captured by models not explicitly allowing for behavioral effects, even if these models allow for variations in unobserved error according to network complexity.

Furthermore, in one of the applications of network formation as mentioned in the introduction, word-of-mouth communication among consumers, it matters for the supplier of the respective product or service to be aware of behavioral effects as found in this study. For instance, consumers have a tendency to talk with isolated peers and they neglect communication benefits from beyond their direct neighbors in their communication decisions. Also, she can exploit the finding that this heuristic behavior is dependent on the complexity of the network environment. 
One of the directions that follow-up studies could take concerns the question in how far the complexity types and behavioral effects we considered are specific for the network context. For example, would the tendency to link creation have an equivalent in other choice settings? Moreover, future experiments could generate further insights in the linking choice process of individuals by concentrating on specific effects from the rich range of heuristic tendencies explored here. 


\section{References}

[1] Bala, V. and S. Goyal (2000), "A Noncooperative Model of Network Formation", Econometrica 68(5), 1181-1229.

[2] Berninghaus, S.K., K.-M. Ehrhart, and M. Ott (2006), "A Network Experiment in Continuous Time: The Influence of Link Costs", Experimental Economics 9(3), 237-251.

[3] Bonner, S.E. (1994), "A Model of the Effects of Audit Task Complexity", Accounting, Organizations and Society 19(3), 213-234.

[4] Broniarczyk, S.M. and J.W. Alba (1994), "Theory versus Data in Prediction and Correlation Tasks", Organizational Behavior and Human Decision Processes 57(1), 117-139.

[5] Brown, M., A. Falk, and E. Fehr (2004), "Relational Contracts and the Nature of Market Interactions", Econometrica 72(3), 747-780.

[6] Callander, S. and C.R. Plott (2005), "Principles of Network Development and Evolution: An Experimental Study", Journal of Public Economics 89(8), 1469-1495.

[7] Camerer, C. (1998), "Bounded Rationality in Individual Decision Making", Experimental Economics 1(2), 163-183.

[8] Charness, G., G.R. Frechette, and J.H. Kagel (2004), "How Robust is Laboratory Gift Exchange?", Experimental Economics 7(2), 189-205.

[9] Charness, G. and M.O. Jackson (2006), "Group Play in Games and the Role of Consent in Network Formation", forthcoming in Journal of Economic Theory.

[10] Corbae, D. and J. Duffy (2007), "Experiments with Network Formation", forthcoming in Games and Economic Behavior.

[11] Deck, C. and C. Johnson (2004), "Link Bidding in Laboratory Networks", Review of Economic Design 8(4), 359-372.

[12] Fehr, E. and K.M. Schmidt (2003), "Theories of Fairness and Reciprocity - Evidence and Economic Applications", in: Dewatripont, M., L.P. Hansen, and S. Turnovsky, Advances in Economics and Econometrics: Theory and Applications, Eighth World Congress of the Econometric Society 1, Cambridge: University Press, 208-257. 
[13] Forbus, K.D. (1993), "Qualitative Process Theory: Twelve Years After", Artificial Intelligence 59(1-2), 115-123.

[14] Frederick, S., G. Loewenstein, and T. O’Donoghue (2002), "Time Discounting and Time Preference: A Critical Review", Journal of Economic Literature 40(2), 351-401.

[15] Gigerenzer, G., P.M. Todd and the ABC Research Group (1999), Simple Heuristics That Make Us Smart, Oxford: University Press.

[16] Gilovich, T., D. Griffin, and D. Kahneman (2002), Heuristics and Biases: The Psychology of Intuitive Judgment, Cambridge: University Press.

[17] Granovetter, M. (1995), Getting a Job: A Study of Contacts and Careers, 2nd ed., Chicago and London: University of Chicago Press.

[18] Hannon, B. (1994), "Sense of Place: Geographic Discounting by People, Animals and Plants", Ecological Economics 10(2), 157-174.

[19] Hauk, E. and R. Nagel (2001), "Choice of Partners in Multiple Two-Person Prisoner's Dilemma Games", Journal of Conflict Resolution 45(6), 770-793.

[20] Hensher, D.A., J.M. Rose, and W.H. Greene (2005), Applied Choice Analysis: A Primer, Cambridge: University Press.

[21] Iacobucci, D. and N. Hopkins (1992), "Modeling Dyadic Interactions and Networks in Marketing", Journal of Marketing Research 29(1), 5-17.

[22] Jackson, M.O. and A. Wolinsky (1996), "A Strategic Model of Social and Economic Networks", Journal of Economic Theory 71(1), 44-74.

[23] Jackson, M.O. and A. Van den Nouweland (2005), "Strongly Stable Networks", Games and Economic Behavior 51(2), 420-444.

[24] Johnson, E.J. and J.W. Payne (1985), "Effort and Accuracy in Choice", Management Science 31(4), 395-414.

[25] Kahneman, D. (2003), "Maps of Bounded Rationality: Psychology for Behavioral Economics", The American Economic Review 93(5), 1449-1475. 
[26] Kirchsteiger, G., M. Niederle, and J. Potters (2005), "Endogenizing Market Institutions: An Experimental Approach", European Economic Review 49(7), 1827-1853.

[27] Levinthal, D.A. and J.G. March (1993), "The Myopia of Learning", Strategic Management Journal 14(special issue winter), 95-112.

[28] Loasby, B.J. (1976), Choice, complexity and ignorance, Cambridge: University Press.

[29] McFadden, D. (2001), "Economic Choices", The American Economic Review 91(3), 351-378.

[30] McKelvey, R.D. and T.R. Palfrey (1995), "Quantal Response Equilibria for Normal Form Games", Games and Economic Behavior 10(1), 6-38.

[31] Perrings, C. and B. Hannon (2001), "An Introduction to Spatial Discounting", Journal of Regional Science 41(1), 23-38.

[32] Shafir, E., I. Simonson, and A. Tversky (1993), "Reason-Based Choice", Cognition 49(1-2), 11-36.

[33] Simon, H.A. (1979), "Rational Decision Making in Business Organizations", The American Economic Review 69(4), 493-513.

[34] Swait, J. and W. Adamowicz (2001), "The Influence of Task Complexity on Consumer Choice: A Latent Class Model of Decision Strategy Switching", Journal of Consumer Research 28(1), $135-148$.

[35] Van den Bulte, C. and R.K. Moenaert (1998), "The Effects of R\&D Team Co-location on Communication Patterns among R\&D, Marketing, and Manufacturing", Management Science 44(11), S1-S18.

[36] Wellman, B., J. Salaff, D. Dimitrova, L. Garton, M. Gulia, and C. Haythornthwaite (1996), "Computer Networks as Social Networks: Collaborative Work, Telework, and Virtual Community", Annual Review of Sociology 22(1), 213-238. 


\section{Appendix A: Instructions}

\section{1 social tradeoff? NO value transferability? NO YES}

In this experiment you are asked to respond to eight choice problems. You can earn points depending on the choices you make in these problems. The total number of points that you have at the end of the experiment determines your monetary payoff.

In each problem, you see a picture of a network in which you and several other nodes are interconnected by links. In order to generate points, you are allowed to change at most one link. You have the following options to do this: (1) you can delete one link that already exists between you and any other node, (2) you can create one link between you and any other node if there is not yet any link between you and this node, or (3) you can choose not to change anything.

You can determine the number of points you receive due to your choice for a specific problem, as follows. For each node you are directly linked with (we call such a node a neighbour) you obtain points. For each path that links you to some other node you obtain points. However, there is also some cost associated with being connected: the number of points you receive for each of your direct neighbours equals 10 divided by two components: (i) the number of direct neighbours you have, and (ii) the number of direct neighbours this neighbour has. the number of points you receive for each path that links you to some other node equals 10 divided by three components: (i) the number of direct neighbours you have in the network, (ii) the number of direct neighbours this other node has in the network, and (iii) the square of the number of direct neighbours that any of the further nodes on the path between you and the other node has in the network.

[example, see Section 6.3]

After each of the eight problems, the number of points that you earned will be reported.

Note that there are no real people behind the other nodes in a network: you are the only one able to change a link and earn points by this.

At the end of the experiment, points are exchanged for euros in the following way:

amount in euros you receive $=4+\mathbf{0 . 4} 0.3$ (total number of points that you earned - 33.68 45.17).

\section{2 social tradeoff? YES value transferability? NO YES}

In this first part you are asked to respond to eight choice problems. You can earn points depending on the choices you make in these problems. Moreover, your choices can also generate points for the other participants in the room. The total number of points that you have at the end of the experiment determines your monetary payoff.

In each problem, you see a picture of a network in which you and several other nodes are interconnected by links. In order to generate points, you are allowed to change at most one link. You have the following options to do this: (1) you can delete one link that already exists between you and any other node, (2) you can create one link between you and any other node if there is not yet any link between you and this node, or (3) you can choose not to change anything.

You can determine the number of points you receive due to your choice for a specific problem, as follows. For each node you are directly linked with (we call such a node a neighbour) you obtain points. For each path that links you to some other node you obtain points. However, there is also some cost associated with being connected: the number of points you receive for each of your direct neighbours equals 10 divided by two components: (i) the number of direct neighbours you have, and (ii) the number of direct neighbours this neighbour has. the number of points you receive for each path that links you to some other node equals 10 divided by three components: (i) the number of direct neighbours you have in the network, (ii) the number of direct neighbours this other node has in the network, and (iii) the square of the number of direct neighbours that any of the further nodes on the path between you and the other node has in the network.

[example, see Section 6.3]

After each of the eight problems, the number of points that you earned will be reported.

The other nodes in the choice problems receive points in the same way as you do. There are no real people behind these nodes and you are the only one able to change a link in a network. However, the points that the other nodes receive due to your choices do have a consequence for the other participants in this room. In fact, these points will be divided equally among them.

[example continued, see Section 6.3]

The number of points that you generated for the other participants will also be reported after each problem.

At the end of the experiment, points are exchanged for euros in the following way:

amount in euros you receive for this first part $=4+\mathbf{0 . 0 6} 0.07$ (total number of points that you earned in this first part - 265.51320 .63$)$.

The choices you made in the first part do not influence the payoffs in this part and the choices you will make in this part do not influence the payoffs in the previous part. 
In this second part you are asked to respond to eight choice problems. You can earn points depending on the choices you make in these problems. Moreover, your choices can also generate points for the other participants in the room. The total number of points that you have at the end of the experiment determines your monetary payoff.

At the end of the experiment, points are exchanged for euros in the following way:

amount in euros you receive for this second part $=0.5+\mathbf{0 . 0 3} 0.035$ (total number of points that you earned in this second part - 265.51 320.63).

\subsection{Example}

\subsection{1 social tradeoff? NO value transferability? NO}

For example, in the above network [Figure 1] you have two direct neighbours: a and d. For neighbour a you get 10 points divided by 2 (since you have two direct neighbours) divided by 3 (since a has three direct neighbours). For neighbour d you get 10 points divided by 2 (since you have two direct neighbours) divided by 2 (since $\mathrm{d}$ has two direct neighbours). In total you therefore receive $10 / 6+10 / 4=25 / 6$ points in this example.

\subsection{2 social tradeoff? NO value transferability? YES}

For example, in the above network [Figure 1] there are two paths between you and c. For the path via a and b you get 10 points divided by 2 (since you have two direct neighbours in the network) divided by $3 * 3$ (since a has three direct neighbours in the network) divided by $2 * 2$ (since b has two direct neighbours in the network) divided by 1 (since $\mathrm{c}$ has one direct neighbour in the network). For the path via d, a, and b you get 10 points divided by 2 (since you have two direct neighbours in the network) divided by $2 * 2$ (since $\mathrm{d}$ has two direct neighbours in the network) divided by $3 * 3$ (since a has three direct neighbours in the network) divided by $2 * 2$ (since b has two direct neighbours in the network) divided by 1 (since $\mathrm{c}$ has one direct neighbour in the network). In total you therefore receive 10/72 $+10 / 288=25 / 144$ points for the paths between you and c. In the same way you get $10 / 36+10 / 144$ points for the paths between you and b, $10 / 6+10 / 24$ points for the paths between you and a and $10 / 4+10 / 36$ points for the paths between you and d. In total you therefore receive $775 / 144$ points in this example.

\subsection{3 social tradeoff? YES value transferability? NO}

For example, in the above network [Figure 1] you have two direct neighbours: a and d. For neighbour a you get 10 points divided by 2 (since you have two direct neighbours) divided by 3 (since a has three direct neighbours). For neighbour d you get 10 points divided by 2 (since you have two direct neighbours) divided by 2 (since d has two direct neighbours). In total you therefore receive $10 / 6+10 / 4=25 / 6$ points in this example.

[continued] In the example above, node c has one direct neighbour: b. Therefore, she receives 10 points divided by 1 (since you have one direct neighbour) divided by 2 (since b has two direct neighbours), which implies 5 points. In the same way, node $\mathrm{b}$ gets $10 / 2+10 / 6=20 / 3$ points, node a gets $10 / 6+10 / 6+10 / 6=5$ points, and node $\mathrm{d}$ gets $10 / 4$ $+10 / 6=25 / 6$ points. In total therefore $5+20 / 3+5+25 / 6=125 / 6$ points will be divided equally among the other participants in the room in this example.

\subsection{4 social tradeoff? YES value transferability? YES}

For example, in the above network [Figure 1] there are two paths between you and c. For the path via a and b you get 10 points divided by 2 (since you have two direct neighbours in the network) divided by $3 * 3$ (since a has three direct neighbours in the network) divided by $2 * 2$ (since b has two direct neighbours in the network) divided by 1 (since $\mathrm{c}$ has one direct neighbour in the network). For the path via d, a, and b you get 10 points divided by 2 (since you have two direct neighbours in the network) divided by $2 * 2$ (since $\mathrm{d}$ has two direct neighbours in the network) divided by $3 * 3$ (since a has three direct neighbours in the network) divided by $2 * 2$ (since b has two direct neighbours in the network) divided by 1 (since c has one direct neighbour in the network). In total you therefore receive 10/72 $+10 / 288=25 / 144$ points for the paths between you and c. In the same way you get $10 / 36+10 / 144$ points for the paths between you and b, $10 / 6+10 / 24$ points for the paths between you and a and $10 / 4+10 / 36$ points for the paths between you and d. In total you therefore receive $775 / 144$ points in this example.

[continued] In the example above, there is one path between nodes a and c. Therefore, c receives 10 points divided by 1 (since $\mathrm{c}$ has one direct neighbour) divided by $2^{*} 2$ (since b has two direct neighbours) divided by 3 (since a has three direct neighbours $)=5 / 6$ points for the paths between her and a. In the same way c gets $10 / 2$ points for the path between her and b, 10/72 + 10/288 points for the paths between her and d, and 10/72 $+10 / 288$ points for the paths between her and you. In total $\mathrm{c}$ therefore receives $445 / 72$ points. In the same way node $\mathrm{b}$ gets $10 / 2+10 / 6+10 / 36+$ $10 / 144+10 / 36+10 / 144$ points, node a gets $10 / 12+10 / 6+10 / 6+10 / 24+10 / 6+10 / 24$ points, and node $\mathrm{d}$ gets $10 / 72+10 / 288+10 / 36+10 / 144+10 / 6+10 / 24+10 / 4+10 / 36$ points. In total therefore $445 / 72+265 / 6+20 / 3$ $+775 / 144=2995 / 48$ points will be divided equally among the other participants in the room in this example. 


\section{Appendix B: Choice Problems}

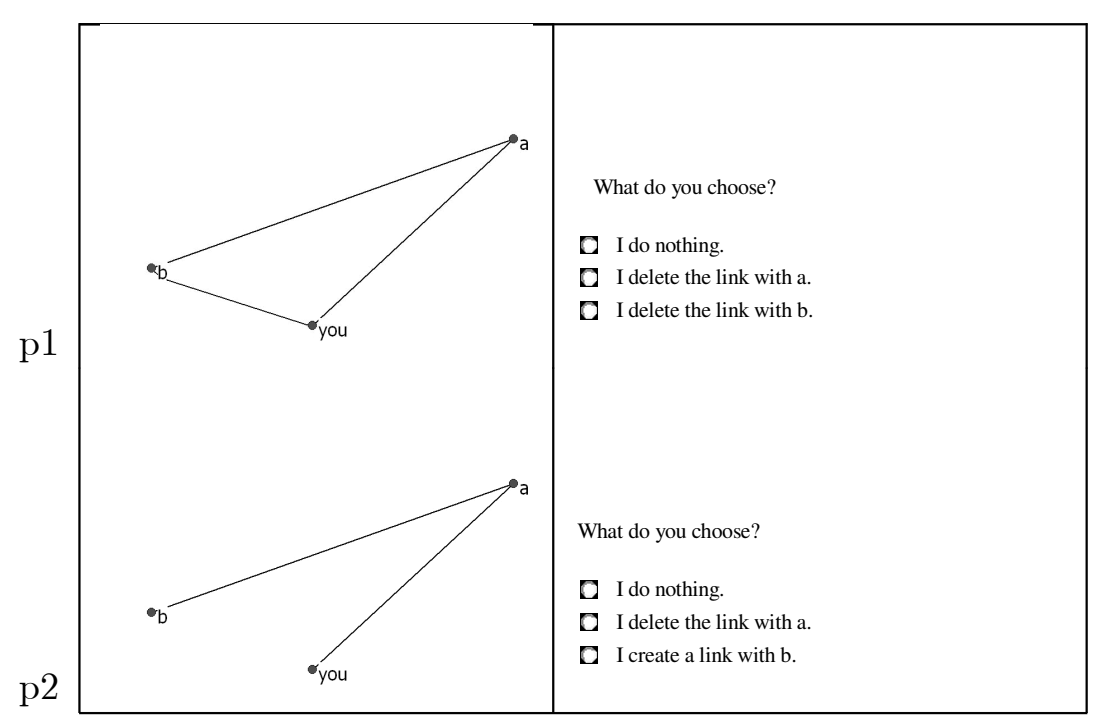

Table 6: Practice Rounds

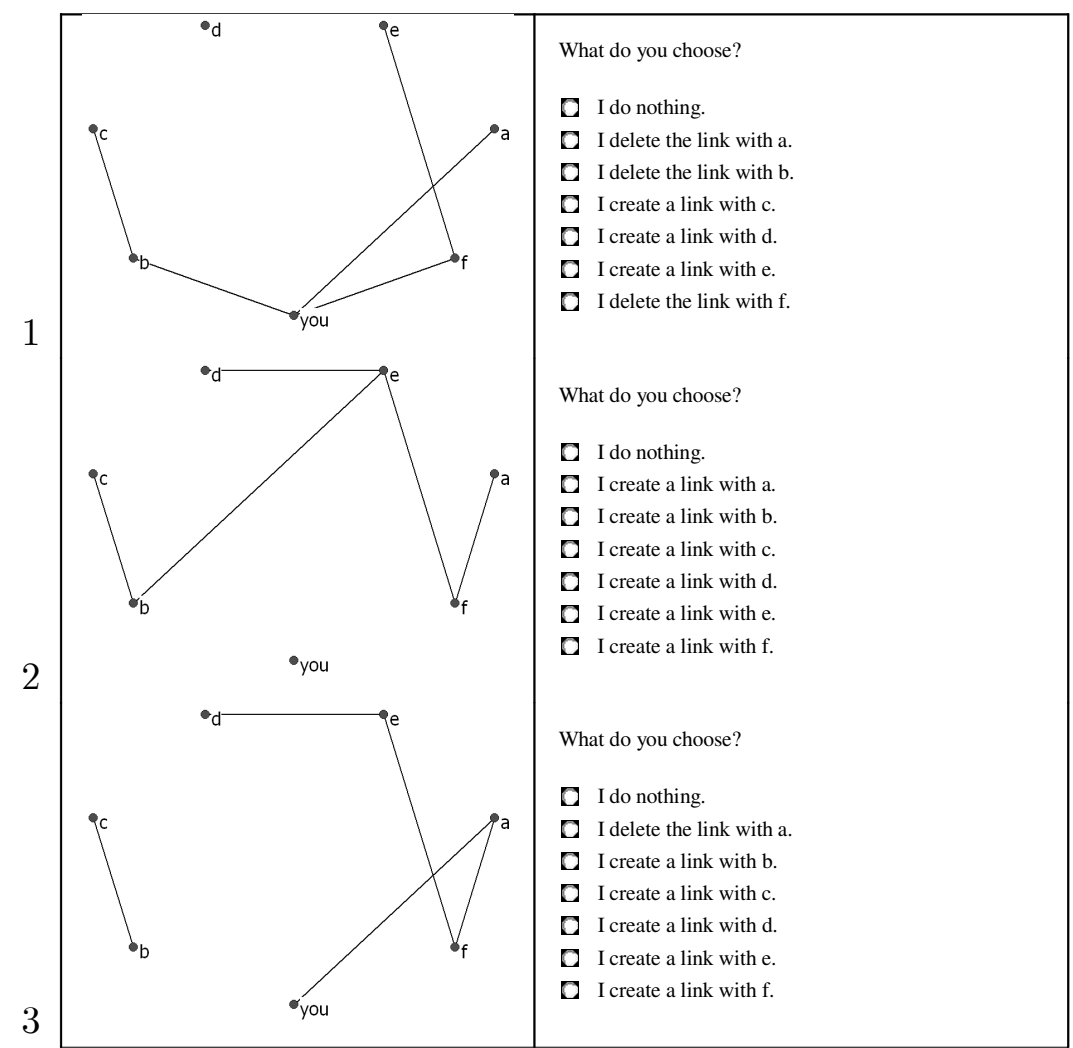

Table 7: Choice Problems 1 - 3 


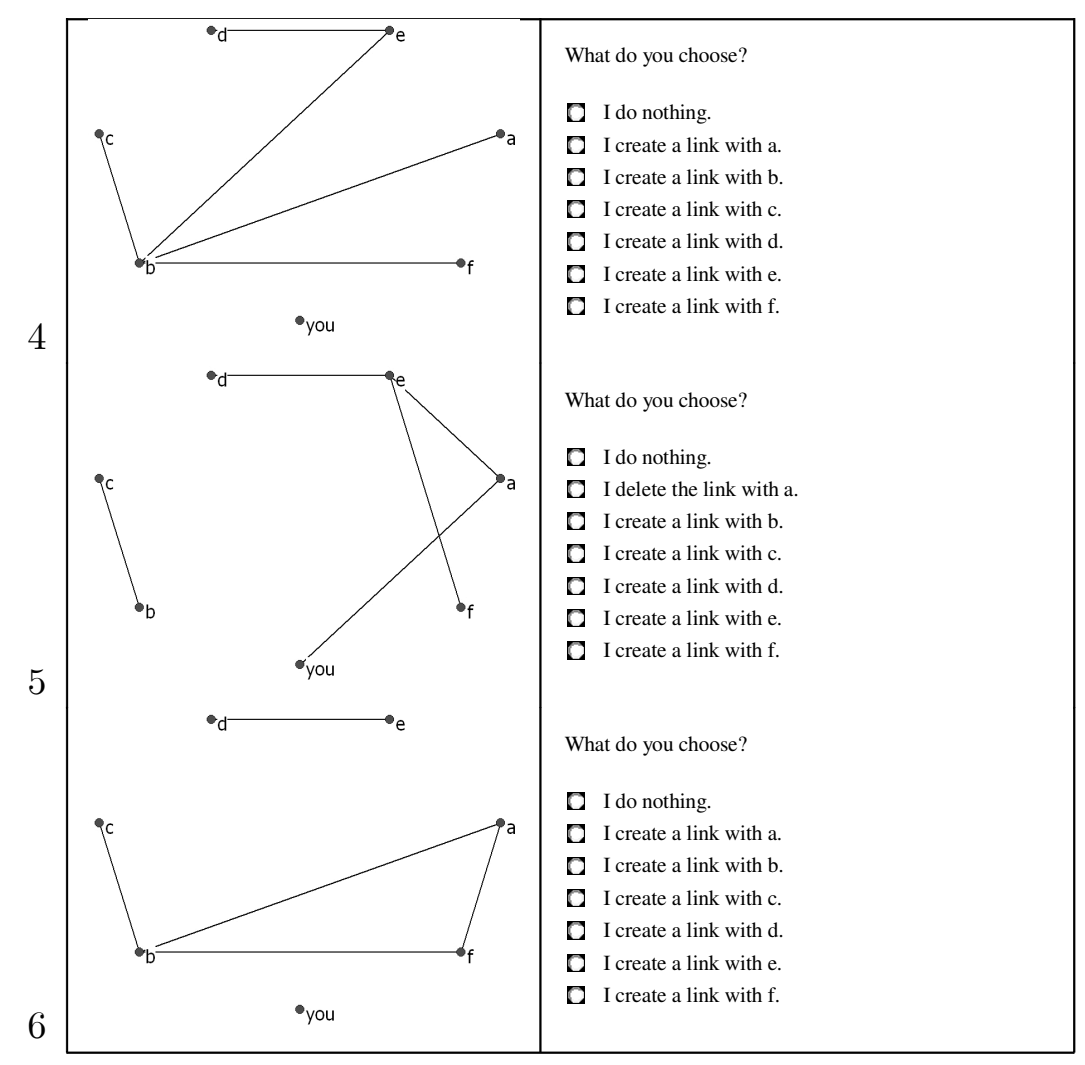

Table 8: Choice Problems 4 - 6

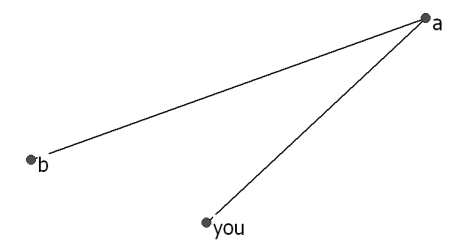

What do you choose?

D I do nothing. This means that I earn 5 points and 15 points will be divided equally among the other participants in this room.

D I delete the link with a. This means that I earn 0 points and 20 points will be divided equally among the other participants in this room.

D I create a link with $\mathrm{b}$. This means that I earn 5 points and 10 points will be divided equally among the other participants in this room.

Figure 3: Illustration Payoff Information 


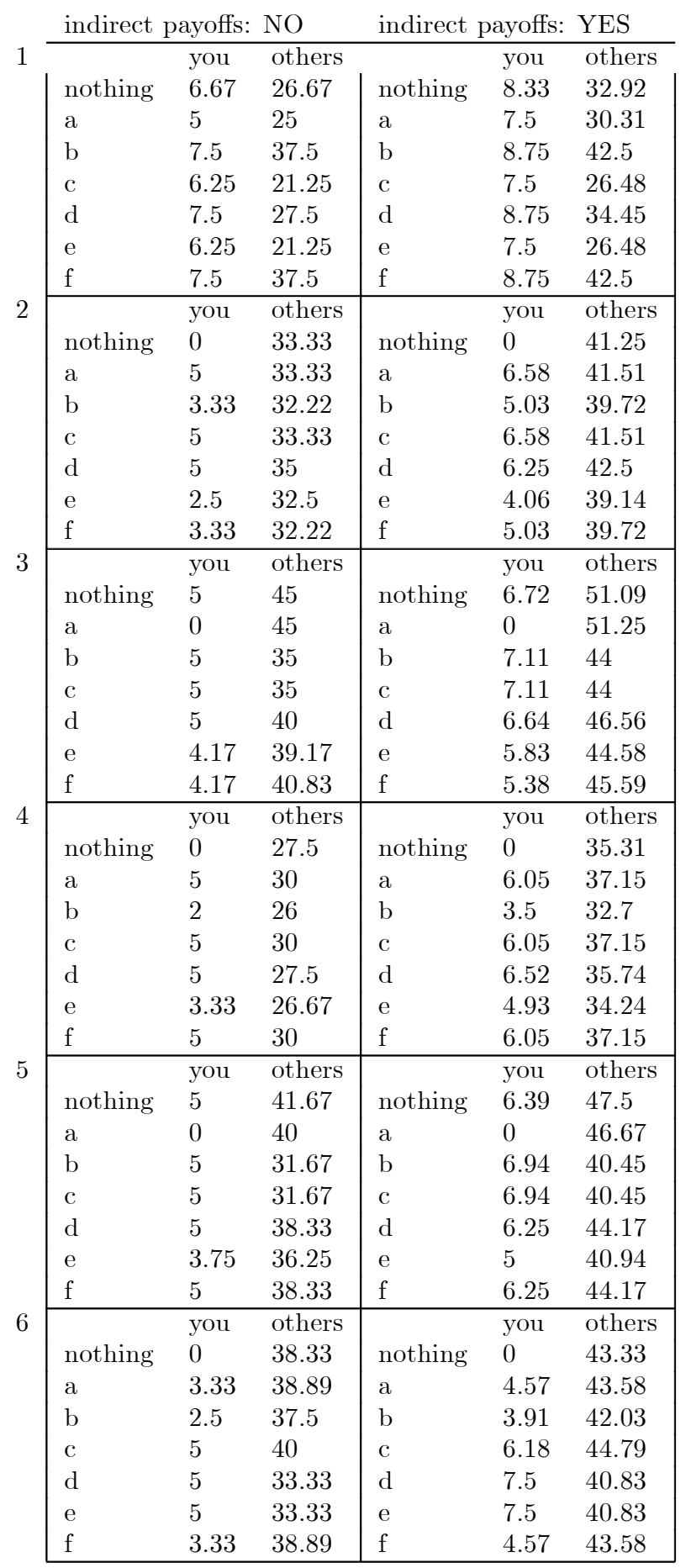

Table 9: Payoffs Choice Problems 


\section{Appendix C: Descriptives}

1. Duration: average 40,2 min., stand. dev. $14,8 \mathrm{~min}$.

2. Almost all participants tried to earn as much as possible, whereas 17 subjects indicated other goals: best choices (6), fun / interest (2), optimal own payoffs and not too bad payoffs for the others (4), optimal own payoffs and minimal payoffs for the others (1), structural goals (4).

3. In the first choice problem [practice round], participants chose as follows: at random: 1, by calculation: 60 , by intuition: 34 , using a rule: 13 , namely connect to the one with the least neighbors / shortest paths (13), otherwise: 6 , namely mix of intuition and calculation (5), mistake in understanding instructions at first (1).

4. Thereafter, did participants change their strategies? No: 67 , for the strategy was good or convenient and the problems were similar, yes: 47 , switch (more) to calculation (12), intuition / experience (22), or rule mentioned in descriptive 3 (11), or consider other participants more (2).

5. In conditions social and both, did participants take into account the points created for other participants? 36 did not, since they didn't think about it (4), didn't care about it (16), didn't know how (5) or didn't like the effort (11), 20 did, where they (conditionally) maximized $(>=8)$ or minimized $(>=3)$ the points for the others, $>=2$ participants seem not to understand that dividing among other participants does not include yourself.

6. Strategies in the second part of conditions social and both: (conditionally) maximizing payoffs for the others (25), choosing not too badly for the others (7), (conditionally) minimizing payoffs for the others (6), trying to repeat part 1 (8), unclear (10).

7. Strategic considerations in conditions social and both? No: 18 , since they didn't think about it (7), thought that the other participants wouldn't care (5), the other participants are outside control (4), or it would be too difficult (2), yes, but did not influence choices: 9, yes, hoping for a favourable group: 5, or expecting an unfavourable group: 2, yes, unclear how: $22(>=5$ of these seem not to understand that this question is about the others creating points for you and not about you creating points for the others).

8. Difficulties were in the following fields: calculation: 33 , choice complexity: 34 , instructions: 27 , equivalent options: 5, none: 16 .

9. Further remarks: interesting / nice: 12, want to know more about the experiment: 10, confirming what was said before: 5, suggestions: 10 .

10. Age: average 22,5 yrs., stand. dev.: 3,4 yrs.

11. Male: 48 , female: 66 .

12. Dutch: 40, German: 43, Chinese: 9, other: 22 .

13. Faculty of Economics \& Business Administration: 90, other: 24.

14. 90 participants did not participate in a similar experiment before; 24 did.

15. 112 participants would like to participate in future experiments, 2 would not.

16. In conditions social and both: 40 participants did not know any of their fellow session participants, 12 knew one and 4 knew more. 